\title{
Observations on peripheral servo mechanisms in Parkinsonian rigidity
}

\author{
WILLIAM WARD HOFMANN \\ From the Clinical Neurophysiology Laboratory, Department of Neurology, \\ Veterans Administration Hospital, and the Division of Neurology, Stanford University \\ School of Medicine, Palo Alto, California, U.S.A.
}

Among the motor system disorders the clinician is called upon to treat perhaps one of the most challenging and frustrating is still the condition described as Parkinson's disease. Although advances continue in both the medical and surgical aspects of therapy (Cooper, 1956; Doshay, 1954; Meyers, 1942; Spiegel and Wycis, 1958), the exact mechanism responsible for the benefit in each case remains unknown. Further, satisfactory definition of the pathophysiology is not yet available, and no accurate model of the clinical condition has been discovered.

The essential physiological abnormality, whether the patient complains chiefly of stiffness or of tremor, is that there is unusual driving of the motor cells in the anterior funiculi of the spinal cord. Most investigations have centred about possible central sources of such continuous or periodic facilitation (Bucy, 1949; Meyers, 1942; Ward, McCulloch, and Magoun, 1948; Spiegel and Wycis, 1958; Adey, Buchwald, and Lindsley, 1960; Carpenter, Glinsman, and Fabrega, 1957), but recently attention has been directed more peripherally toward the operation of the so-called 'small fibre motor system' and the muscle spindle apparatus (Granit, 1955; Hassler, 1955; Eldred, Granit, and Merton, 1953).

The muscle spindles, length sensors lying in parallel' with the extrafusal fibres, are important regulators of alpha motor cell output (Hunt, 1952; Kuffler, Hunt, and Quillian, 1951). Through a relatively slow pathway the spindles can be 'set' for postural adjustments (Granit, 1955; Merton, 1953) but their phasic sensitivity and feedback characteristics can also be modified through channels operating at alpha velocity (Granit, Pompeiano, and Waltman, 1959a, b; Rutledge and Haase, 1961). Malfunctioning in components of the small fibre system has lately been held accountable for some of the features of Parkinson's disease (Hassler, 1955; Rushworth, 1959), though the technique of direct recording from gamma efferent or afferent fibres is not yet available in humans.
The purpose of these experiments was to study, by indirect methods, the activity of the muscle spindles in patients afflicted with the rigidity of Parkinson's disease and to compare the findings with those in normal subjects.

\section{METHODS}

Observations were made on eight subjects with typical Parkinsonian rigidity and an equal number of normals. Four of the afflicted subjects were studied while on medication, four after drug withdrawal, and it was observed that full doses of drugs made no difference in the results.

The first group of experiments was performed with a technique very similar to that described by Merton (1951). In each case, the right arm was used. Electromyographic recording was carried out by means of concentric needle electrodes inserted into the adductor of the right thumb. In some cases, the median nerve was blocked with local anaesthetic at the wrist in order to silence the opponens pollicis. The thumb was attached to a strain gauge and was held tightly against the plane of action of the adductor. The strain gauge output was monitored with DC amplification and filmed on the second beam of the oscilloscope. The patient was provided with a microammeter null indicator in parallel with the output of the strain gauge and could maintain constant tension by observing the microammeter himself. It was always made clear to the patient that a constant thumb posture was required once the specified degree of tension was attained. Percutaneous stimulating electrodes were placed over the right ulnar nerve, either at the elbow or at the ulnar side of the wrist, and shocks of 600 microseconds' duration were applied at one or two seconds' intervals while the patient maintained a fixed tension. The E.M.G. and ergographic displays were first recorded simultaneously from a dual beam oscilloscope on continuously moving film and later, in six afflicted subjects, with multiple exposures in a single frame. For the superimposed tracings, the sweep was triggered 80 to 100 milliseconds before each stimulation.

The first group of studies concerned the silent period in the thumb adductor after stimulation of the ulnar nerve while the patient maintained a fixed baseline con- 
traction. The length and intensity of alpha motoneurone 'shutdown' in response to a twitch was compared in normal and afflicted subjects.

The second group of observations was designed to study the effects of central modification of proprioceptive feedback. In these tests the Jendrassik manoeuvre was used in both normal and afflicted subjects, and the results were measured in terms of visible and palpable facilitation of the quadriceps reflex.

The last group of experiments was designed to evaluate the availability of the peripheral feedback mechanism in paralysis agitans. Resting bias could, perhaps, be estimated from the silent period experiments, but passive elongation of the peripheral sensor would show whether it could fire in response to peripheral events. Here, the thumb adductor was simply stretched passively by the investigator.

\section{RESULTS}

In both normal and afflicted subjects the silent period was studied hundreds of times. This well-known phenomenon was found in normal subjects, as it has been in previous studies (Merton, 1951; Merton, 1953), to be quite constant in duration and intensity regardless of the initial tension or of the evoked twitch tension. In fact, barely supra-threshold shocks to the ulnar nerve were adequate to evoke the cessation of mctoneurone firing, and the slightest visible twitch of the thumb adductor was all that was required. Examples of responses in normal subjects are seen in Figure 1.

In patients with the rigidity of Parkinsonism the results were quite different. As shown in serial recording on moving film (Figs. 2A, B), the addition of a twitch to a sustained voluntary contraction often failed entirely to evoke a silent period. This complete absence of the spindle pause was observed regularly in only three cases, but in all afflicted subjects with significant clinical rigidity the lulls in motor activity after a twitch averaged less than normal. When each twitch was studied separately on moving film normal silent periods were occasionally seen, even in the most rigid patients, but superposition of repeated twitches on one film frame consistently revealed defects as shown in Figures $2 \mathrm{C}$ and 2D. A more statistical evaluation was obtained by comparing 200 evoked responses in a group of eight afflicted against an equal number in eight normal subjects. Several parameters could be compared, but it was felt that weighing of the greatest defect (no suppression of motor discharge) might be most meaningful. Twenty-three total failures of the silent period were observed in the afflicted series and five in the normals. This relationship has significance at the $\mathbf{P}=0.01$ level.

The second group of experiments involved the Jendrassik manoeuvre. The test, performed by ccmparing the amplitude of the knee jerk before and during the classical hand manoeuvre, produced obvicus augmentation in all afflicted patients in this study, as well as in numerous other cases in the wards, suggesting that the lower motor unit and its peripheral length sensor are quite accessible to some facilitatory influences driving the annulospiral apparatus, even in the face of Parkinsonian rigidity. This finding is at variance with results reported by Jung and Hassler (1960).

The last portion of the study was concerned with the response of the lower motor neurone (as recorded
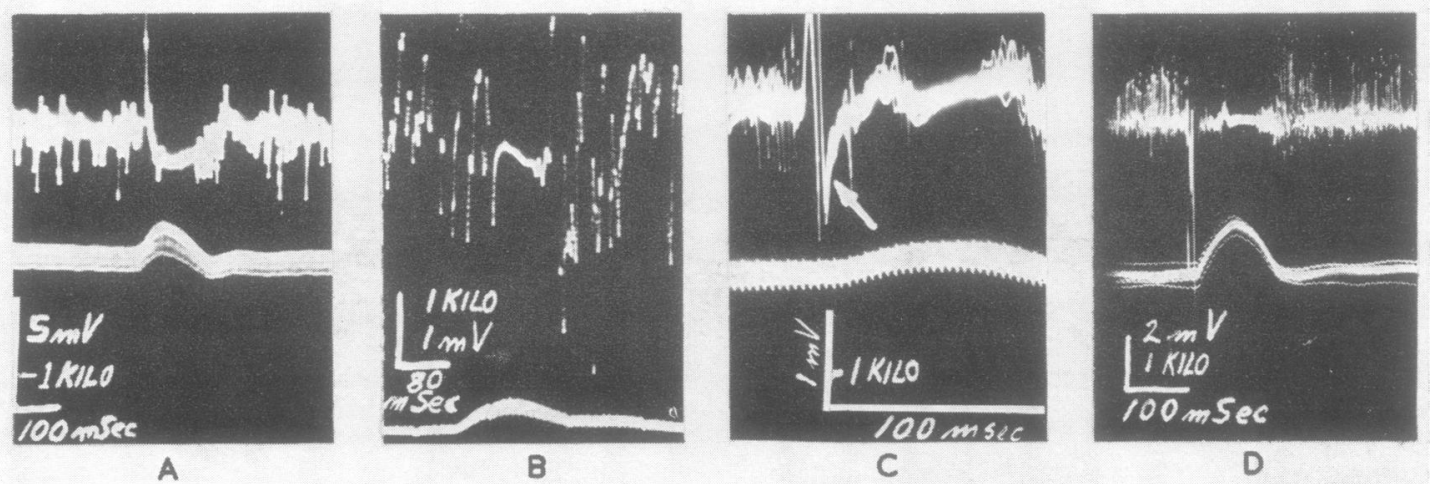

FIG. 1. Silent periods in normal subjects. Stimulating shocks recorded as large E.M.G. artefacts (upper trace), just at foot of each twitch (lower trace). A and B, single twitches from moving film records. Note that even very weak twitch in $B$ is followed by a normal silent period. $C, 10$ consecutive sweeps superimposed at high sweep speed. D, 15 sweeps at slower speed. Median nerve blocked at wrist in C and D. Antidromic block seen in $C$ just after shock. Artefact at arrow (Merton, 1951). 

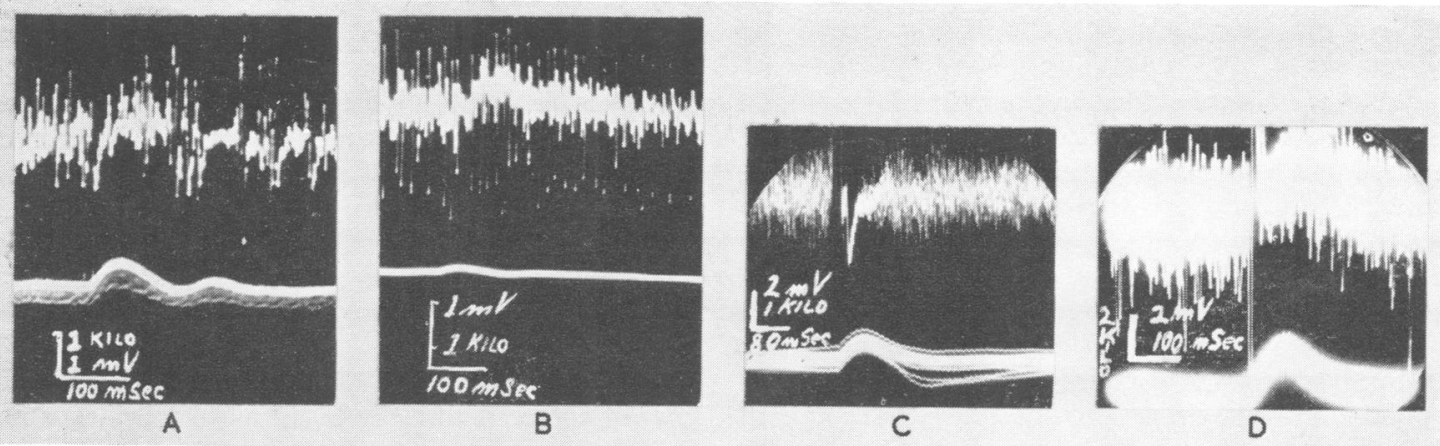

FIG. 2. Silent period defects in patients with Parkinsonian rigidity and little or no tremor. $A$ and $B$, single examples from serial (moving film) recordings in two patients. Note that, though the twitch was not followed by a silent period in $A$, tremor oscillation thereafter was associated with definite quiescence. $C$ and $D$ are single-frame exposures of 10 traces each in two subjects. C shows considerable shortening of the silent period (compare with Fig. 1C). D shows the major defect recorded with this technique in a rigid patient. Median nerve blocked at wrist in $B, C, D$.

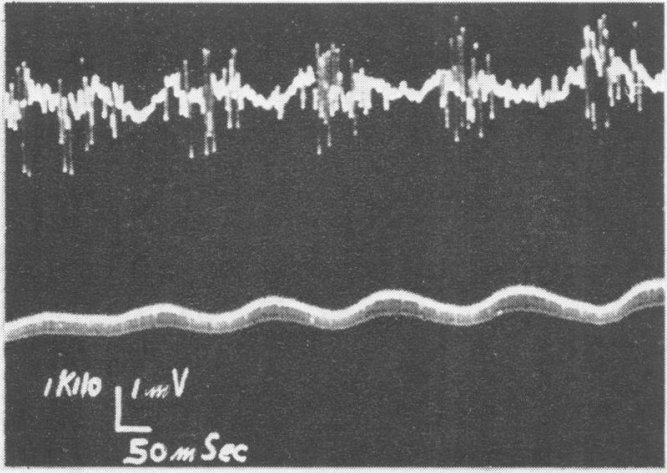

A

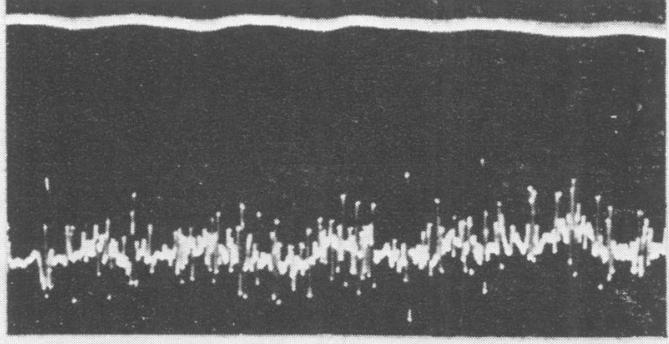

B

fIG. 3. $A$ and $B$, consecutive film strips in patient with moderately severe rigidity and tremor. $A$, resting tremor volleys. $B$, regularization and increase in alpha cell output by stretching muscle. Thumb adductor passively elongated by examiner. Calibrations same for both records. Ergograph beam displaced in $B$ by traction on patient's hand to stretch thumb adductor. in the E.M.G.) while proprioceptive input was modified more peripherally. Here the stretch reflex was studied in afflicted subjects and compared with the normal. While the patient was relaxed the thumb adductor was passively stretched by the examiner. Figures $3 \mathrm{~A}$ and $3 \mathrm{~B}$ show the response of the thumb adductor in a patient with severe rigidity and slight tremor when the muscle was quickly stretched. The increasing input from the spindle was clearly capable of driving the anterior horn cell.

\section{DISCUSSION}

Some observers suggest that Parkinsonism results from failure of cerebral damping and integrating circuits (Spiegel and Wycis, 1958; Doshay, 1954; Bucy, 1949); others indicate that there is a paralysis (Jung and Hassler, 1960; Hassler, 1955) or hyperactivity (Rushworth, 1959) of peripheral muscle sensors. Some difficulty may arise with each of the several interpretations, and it may be easier to consider the one abnormality common to all cases.

The fundamental problem in the case of rigidity is sustained bombardment of agonist and antagonist alpha neurones in the cord, such that the muscles receive a nearly constant discharge regardless of their length and are equally driven on both sides of a joint. While the abnormal driving of alpha cells must stem partly from descending signals, the final form of the clinical disability may depend largely on what is happening at the same time to the peripheral sensors. A growing body of evidence indicates that peripheral servo mechanisms play a major role in governing the output of the alpha cells (Eldred, 1960; Granit, 1955; Hunt, 1952), and the fact that the sensors are themselves under the 
control of central structures is clearly established (Eldred et al., 1953; Stern and Ward, 1960).

What needs explanation in the light of the present experiments is the failure of the annulospiral apparatus to silence a voluntary discharge when a twitch is added. Several possibilities present themselves. First, the continued facilitation of the anterior horn cell pool might stem directly from increased input from the spindle apparatus which was either 'released' to excessive activity or intensely driven from above so that it could not be turned off. This application fails entirely if one regards the spindle as a differential length indicator, for the more highly such a sensor be 'tuned' the more sensitive it becomes to changes in the main muscle, and the silent period should certainly be preserved or even exaggerated.

Indeed, if the spindles are continuously excited, as in decerebrate spasticity, one finds enhancement and irradiation of the stretch reflexes, lengthening and shortening reactions, oscillation in the effector units as clonus, all quite unlike the plastic rigidity of Parkinsonism. Finally, it should be pointed out that we have found normal silent periods routinely in the most spastic extremities.

Secondly, structural damage to the spindle itself might be blamed, but microscopic studies show no alterations of intrafusal structures, and studies of the stretch reflex in rigid patients show clearly that the peripheral spindle apparatus is capable of qualitatively normal function when it is passively elongated.

Thirdly, the abnormality noted in the silent period might result from sustained alpha cell bombardment from above. However, posterior rhizotomy or local muscle infiltration with anaesthetic may decrease rigidity. Suprasegmental driving producing rigidity and rendering anterior horn cells unresponsive to spindle pauses would not be expected to be so vulnerable to such peripheral manipulations. On the other hand, the relief of Parkinsonian rigidity by posterior root section or drug block does not prove that excessive spindle afferent input is responsible for hypertonus of this type, as the root section abolishes powerful driving forces from skin, etc., and the effects of drugs are probably not limited to gamma fibres.

The present findings leave room for a last interpretation, namely, that the spindle apparatus in the rigid patient has lost some of its innervation from above, is somewhat slack, and has lost most of its sensitivity to differences in length between intraand extrafusal structures, particularly when the descending input calls for postural fixations. While phasic impulses descending at alpha velocity to the alpha and gamma motoneurones may arrive at both in normal fashion, the tonic sensitivity of the peri- pheral length sensor is so low that the unit has lost its ability to indicate correspondence of extra- and intrafusal length, and the muscle may be regulated chiefly by changes in tension. A great number of central as well as peripheral influences on the cord alpha unit may now run unchecked, and simultaneous bombardment of agonist and antagonist units will occur because of the failure in reciprocal inhibitory activity which is one function of the spindle apparatus.

It is necessary to add, however, that the postulated loss of some or all tonic bias on the spindles is not at all the same as de-efferentation, for we have shown that the spindles can be called up to normal sensitivity even in the very rigid patient. Furthermore, de-efferentation should lead to total paralysis of all those modes of motor activity which utilize the spindle loop to drive the alpha cell, but the rigid patient has only relative disability in this regard, and he does not show the degree of motor ataxia which would be expected if only the alpha modes of activation were left. Voluntary motor activity, in view of present findings, is simply delayed in the rigid patient until the spindles can be 'tightened up' to afford drive to synergistic and inhibition to antagonistic alpha neurones. Resting hypertonus is then thought to arise because the descending postural signals, which may use the spindle simply as a null indicator, hit alpha neurones which are no longer 'informed' when their muscle cells have reached proper length.

The experiments relating to the stretch reflexes in afflicted subjects indicate that the cord motor units in this disorder can be altered from the periphery in such a manner as to suggest that the sensor apparatus itself is fully operational, though its resting sensitivity is apparently low. It was not possible to quantitate and compare in normal and afflicted subjects the amount of passive stretch necessary to call up afferent discharge from spindles, but no gross difference was observed.

Since the method used here is, at best, quite indirect, the correctness of the interpretation depends upon control of those variables which would most interfere. The possibility, for example, that defective electromyographic shutdown after a twitch simply reflected the steady 'noise' of other rigid muscles is rendered unlikely by the results after median nerve block at the wrist. The detailed explanations to the subjects regarding the fixity of postural contraction tended to minimize the amount of phasic activity which might interfere. The unavoidable variations in the degree of 'resting' tension from case to case probably added little in the way of error, since it has been shown (Merton, 1951) that this is of little importance, providing a visible twitch is added. The 
results in Fig. 3 (stretch reflex) could, conceivably, be the result of touch stimulation, but the appearance of occasional normal-appearing silent periods in this and other subjects also supports the idea that the spindle apparatus can be called temporarily into the normal sensitivity range.

\section{CONCLUSIONS}

These data are interpreted to mean that defective muscle spindle bias may underlie the rigidity of Parkinson's disease. Comparison between normal and afflicted subjects reveals findings in the rigid patients which are felt not to be the result of recording or other artefacts.

\section{SUMMARY}

Measurements of the silent period in the thumb adductor have been used to evaluate the part played by the alpha motor cells and their servo mechanisms, respectively, in the production of Parkinsonian rigidity. In the interest of simplification, the central lesions associated with the disorder have been neglected except as they may modify the net output of the last stage in the motor apparatus. These investigations have provided some evidence which suggests that Parkinsonian rigidity may be associated with defective bias on muscle length sensors.

This work was supported by United States Public Health Service grant no. B-2602.
REFERENCES

Adey, W. R., Buchwald, N. A., and Lindsley, D. F. (1960). Electroenceph. clin. Neurophysiol., 12, 21.

Bucy, P. C. (Ed.) (1949). The Precentral Motor Cortex, 2nd ed. University of Illinois Press, Ưrbana.

Carpenter, M. B., Glinsman, W., and Fabrega, H. (1957). Neurology, $8,352$.

Cooper, I. S. (1956). Surgical Therapy of Extrapyramidal Disorders: St. Barnabas Symposium. Williams \& Wilkins, Baltimore.

Doshay, L. J. (1954). Parkinsonism and its Treatment, p. 133 Lippincott, Philadelphia.

Eldred, E. (1960). "Posture and locomotion" in Handbook of Physiology, ed. J. Field, Ch. 41, Sect. I: Neurophysiology, Vol. 2, pp. 1067-1088. Williams \& Wilkins, Baltimore.

—, Granit, R., and Merton, P. A. (1953). J. Physiol. (Lond.), 122, 498.

Granit, R. (1955). Receptors and Sensory Perception. Yale University Press, New Haven.

_, Pompeiano, O., and Waltman, B. (1959a). J. Physiol. (Lond.), $147,385$.

(1959b), Ibid., 147, 399.

Hassler, R. (1955). Proc. 2nd Internat. Congr. Neuropath, London, 1955, Pt. I, p. 29. Excerpta medica, Amsterdam.

Hunt, C. C. (1952). J. Physiol. (Lond.), 117, 359.

Jung, R., and Hassler, R. (1960). "The extrapyramidal motor system" in Handbook of Physiology, ed. J. Field, Ch. 35, Sect. I; Neurophysiology, Vol. 2, pp. 863-927. Williams \& Wilkins, Baltimore.

Kuffler, S.W., Hunt, C.C., and Quillian, J.P. (1951). J. Neurophysiol., $14,29-54$

Merton, P. A. (1951). J. Physiol. (Lond.), 114, 183.

- (1953). "Speculations on the Servo Control of Movement." In Wolstenholme, G. E. W. (Ed.) The Spinal Cord: Ciba Foundation Symposium, pp. 247-260. Churchill, London.

Meyers, R. (1942). Ass. Res. nerv. Dis. Proc., 21, 602.

Rushworth, G. (1959). Cerebral Palsy Bull., 1, No. 7, p. 3.

Rutledge, L. T., and Haase, J. (1961). J. Neurophysiol., 24, 182.

Spiegel, E. A., and Wycis, H. T. (1958). In Pathogenesis and Treatment of Parkinsonism, Fields, W. S. (Ed.). Thomas, Springfield, Illinois.

Stern, J., and Ward, A. (1960). Arch. Neurol. (Chicago), 3, 193.

Ward, A. A., McCulloch, W. S., and Magoun, H. W. (1948). J. Neurophysiol., 11, 317. 